\title{
On the existence of minima in the Skyrme model
}

\section{B. J. Schroers*}

Department of Mathematics, Heriot-Watt University, Edinburgh EH14 4AS, UK

E-mail: ibernd@ma.hw. ac.uki

ABSTRACT: Well-separated Skyrme solitons of arbitrary degree attract after a suitable relative rotation in space and iso-space, provided the orders of the solitons' leading multipoles do not differ by more than two. I summarise the derivation of this result, obtained jointly with Manton and Singer, and discuss to what extent its combination with earlier results of Esteban allows one to deduce the existence of minima of the Skyrme energy functional.

KEYwords: 'L'skyrmions, soliton interactions.

\section{Introduction}

The Skyrme model is a non-linear field theory in which nuclei are modelled by quantum states of classical topological solitons [i] for minimal energy solutions in a field theory which have an associated integer degree or topological charge. The degree is conserved for topological reasons and ensures the stability of the soliton. In the Skyrme model static configurations are maps

$$
U: \mathbb{R}^{3} \rightarrow S U(2)
$$

Points in $\mathbb{R}^{3}$ will be denoted by $x=\left(x_{1}, x_{2}, x_{3}\right)$ and I write $r$ for the Euclidean length $|x|=\sqrt{x_{1}^{2}+x_{2}^{2}+x_{3}^{2}}$. Physically, $U$ combines the pion fields $\pi_{1}, \pi_{2}, \pi_{3}$ and the $\sigma$-field

$$
U(x)=\sigma(x)+i \pi_{a}(x) \tau_{a},
$$

where $\tau_{a}, a=1,2,3$, are the Pauli matrices and the constraint $\sigma^{2}+\pi_{1}^{2}+\pi_{2}^{2}+\pi_{3}^{2}=1$ is imposed. Here and in the following, summation over repeated indices is always implied. The Skyrme energy functional is

$$
E[U]=-\int d^{3} x\left(\frac{1}{2} \operatorname{tr}\left(L_{i} L_{i}\right)+\frac{1}{16} \operatorname{tr}\left(\left[L_{j}, L_{i}\right]\left[L_{j}, L_{i}\right]\right)\right)
$$

${ }^{*}$ Speaker. 
where

$$
L_{i}=U^{\dagger} \partial_{i} U
$$

and $\partial_{i}=\partial / \partial x_{i}$ with $i=1,2,3$. The Euler-Lagrange equation can be expressed in terms of the modified currents

$$
\tilde{L}_{i}=L_{i}-\frac{1}{4}\left[L_{j},\left[L_{j}, L_{i}\right]\right]
$$

and reads

$$
\partial_{i} \tilde{L}_{i}=0
$$

It was already noted by Skyrme that the finite-energy requirement means that the Skyrme fields have to tend to a constant at spatial infinity, thus becoming effectively maps from $S^{3}$ to $S U(2) \simeq S^{3}$ with an associated integer degree. Skyrme identified the degree physically with the baryon number. The first rigorous proof that for finite-energy Skyrme configuration the degree

$$
\operatorname{deg}[U]=-\frac{1}{24 \pi^{2}} \int d^{3} x \epsilon_{i j k} \operatorname{tr}\left(L_{i} L_{j} L_{k}\right)
$$

is an integer was only given fairly recently in $[2]$. As a result the configuration space

$$
\mathcal{C}=\left\{U: \mathbb{R}^{3} \rightarrow S U(2) \mid E[U]<\infty\right\}
$$

is partitioned into sectors $\mathcal{C}_{k}$ consisting of all finite energy configurations of degree $k$. Faddeev's bound [ini]

$$
E[U]>12 \pi^{2}|k|
$$

(the strict inequality follows from [iendin implies the existence of infima

$$
I_{k}=\inf \left\{E[U] \mid U \in \mathcal{C}_{k}\right\} .
$$

The central question of this talk is whether the infima are attained, i.e. whether minima exist in all sectors of the Skyrme model. In the following I call minimal energy solutions of non-vanishing degree Skyrme solitons.

To illustrate quite how little is known rigorously about the existence of Skyrme solitons note that the hedgehog ansatz

$$
U_{H}(x)=\exp \left(i f(r) \hat{x}_{a} \tau_{a}\right)
$$

with the boundary condition $f(0)=\pi$ and $f(\infty)=0$ leads to a minimisation problem for

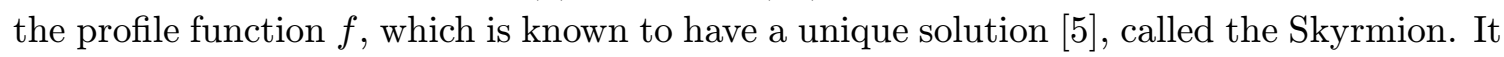

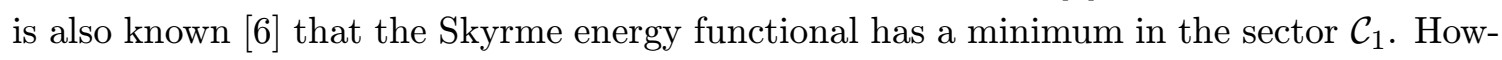
ever, it is still not known whether the minimum is of the spherically symmetric hedgehog form (1).1 1 ). 
Physically, we may think of Skyrme solitons of degree $k>1$ as bound states of Skyrmions. One therefore expects the questions of whether such bound states exist to be related to the existence of attractive forces in the Skyrme model. This expectation is borne out by the analytical work of Esteban in [i6]. Esteban showed that for a suitable class functions

$$
I_{k} \leq I_{l}+I_{k-l}
$$

for all $k, l \in \mathbb{Z}$. Physically this result is only sufficient to ensure threshold bound states - it would be satisfied if for example $I_{k}=C|k|$ for some constant $C$. Esteban went on to show that a minimum exist in $\mathcal{C}_{k}$ provided one assumes the strict inequality

$$
I_{k}<I_{l}+I_{k-l}
$$

for all $k \in \mathbb{Z}-\{0, \pm 1\}$ and $l \in \mathbb{Z}-\{0, k\}$ in the range $|l|+|k-l|<\sqrt{2}|k|$. This result will be referred to as Esteban's theorem in the following discussion. In [2] it was shown that the result still holds if one widens the class of allowed functions, but the inequality (1) 13i) remains a necessary assumption in the proof. In the cases where the infima $I_{l}$ and $I_{k-l}$ are attained by Skyrme solitons, the inequality (1. $\overline{1}$ ij) is equivalent to the existence of attractive forces between those Skyrme solitons. In [i] the existence of attractive forces is studied in detail for Skyrme solitons obeying certain regularity assumptions. In the second part of this talk I review the arguments and results of that paper and in the third part I discuss their relation to Esteban's result.

Before plunging into the technical analysis I should point out that much is known numerically about Skyrme solitons. Numerical searches aided by analytical ansätze suggest

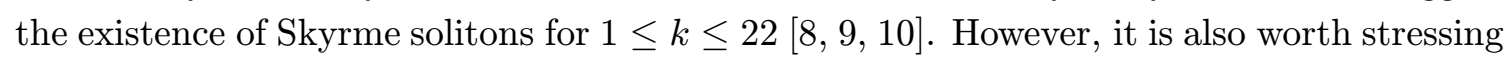
that the existence of classical minima does not ensure the existence of a quantum bound state. This is relevant in the physical application of the Skyrme model because nuclei do not exist for arbitrarily large baryon number. The study of the deuteron as a quantum state of the $k=2$ toroidal Skyrme soliton in [1] 1 in furthermore shows that when such a quantum bound state exists the quantum mechanical matter distribution may be quite different from that of the underlying classical soliton.

\section{The interaction energy of Skyrme solitons}

\subsection{Asymptotics of a Skyrme soliton}

The goal of this subsection is to study the behaviour of Skyrme solitons near spatial infinity. The key step is to think of the 2 -sphere at infinity as a boundary of $\mathbb{R}^{3}$ and to show that the Skyrme equation is regular there. It then follows from a unique continuation argument that non-trivial solutions of the Skyrme equation have a non-trivial expansion near infinity. In other words, Skyrme solitons necessarily have a non-trivial large $r$ expansion in powers of $1 / r$, possibly combined with $\ln r$.

To explain the basic ideas, consider a finite point $x_{0}$ and assume (if necessary redefining $\left.U(x) \rightarrow U\left(x_{0}\right)^{-1} U(x)\right)$ that $U\left(x_{0}\right)=1$. Then we have the expansion

$$
U(x)=1+u(x)
$$


in a neighbourhood of $x_{0}$, with the $2 \times 2$ complex matrix $u$ satisfying the algebraic constraints

$$
u+u^{\dagger}+u u^{\dagger}=0, \quad \operatorname{tr}(u)+\operatorname{det}(u)=0 .
$$

After lengthy algebra, the Skyrme equation can be rewritten

$$
P\left(u, \partial u, \partial^{2} u\right)=Q(u, \partial u)+F(u, \partial u)
$$

where $P$ is linear in the second derivatives $\partial^{2} u$ and contains the Laplace operator $\Delta u$. The functions $Q$ and $F$ crucially do not depend on $\partial^{2} u$ and are, respectively, quadratic and of degree four in $u$. The point of writing the Skyrme equation in this way is that the operator

$$
f \mapsto P\left(u, \partial u, \partial^{2} f\right)
$$

is linear and elliptic near $x_{0}$. If we assume that both $u$ and the currents $L_{i}$ are Hölder

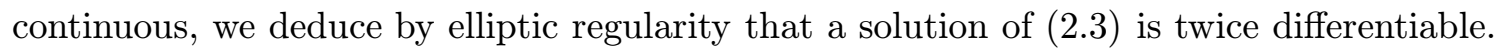
Bootstrapping further, one concludes that any solution is in fact smooth (i.e. $C^{\infty}$ ). The key idea in this procedure is to re-interpret the non-linear Skyrme equation as an elliptic linear equation with coefficients depending on $u$ and $\partial u$.

In order to apply the same idea at spatial infinity, we treat the 2-sphere at spatial infinity as a boundary of $\mathbb{R}^{3}$ and introduce coordinates $(s, \theta, \varphi)$, where $s=1 / r$ and $\theta$ and $\varphi$ are the usual spherical coordinates. Then defining

$$
D_{i}=r \partial_{i}=\frac{1}{s} \partial_{i}
$$

the Euclidean Laplacian takes the form

$$
\Delta=s^{2} \Delta_{b}
$$

with

$$
\Delta_{b}=s^{2} \partial_{s}^{2}+\Delta_{\omega}
$$

where $\Delta_{\omega}$ is the Laplacian of the unit 2-sphere. Now we write $U=1+u$ for large $r$ (that is, for small positive $s$ ) and obtain a ' $b$ '-version of the Skyrme equation

$$
P_{b}\left(u, D u, D^{2} u\right)=Q_{b}(u, D u)+s^{2} F_{b}(u, D u) .
$$

This equation can now be analysed using tools from the theory of so-called $b$-differential operators, see $[\overline{1} \overline{1} \overline{2}, \overline{1}, \overline{1} \overline{3}$. In particular one can use the boundary conditions that $U$ tends to one at $\infty$ and that the currents $L_{i}$ vanish there to solve $\left(\overline{2}_{2}^{2} \overline{8}_{i}^{i}\right)$ iteratively, thus producing an expansion of the solution in powers of $s=1 / r$, with possible factors $\ln r$ in higher order terms. At this stage we have an asymptotic expansion of Skyrme solitons, but do not yet know whether there is a leading non-zero term in the expansion. This can be established with the help of a unique continuation theorem [i] 
The upshot of this chain of arguments, described in detail in [i]i], is that every Skyrme soliton has a leading Lie-algebra valued multipole field

$$
u_{M}(x)=i \tau_{a} \sum_{m=-M}^{M} \frac{4 \pi}{2 M+1} Q_{M m}^{a} \frac{Y_{M m}(\theta, \varphi)}{r^{M+1}},
$$

where $Y_{M m}$ are the usual spherical harmonics on $S^{2}$. In accordance with the usual nomenclature we refer to the multipole field $(\overline{2} . \overline{9})$ as a $2^{M}$-pole and call $M$ the order of the multipole. The leading multipole moments $Q_{M m}^{a}$ are independent of the location of the Skyrme soliton, and are acted on naturally by rotations and iso-rotations. They are crucial for the calculations in the following section. Note that it was already shown in [i] $\left[\begin{array}{l}\overline{1} \\ \overline{1}\end{array}\right]$ that the leading multipole cannot be a monopole. The Skyrmion field (1).11i) is known to have an iso-triplet of dipoles as leading multipoles. For other Skyrme solitons, too, the leading multipoles have been investigated to some extent. The leading multipole of the largest known order is an octupole (i.e. multipole order 3) which is believed to arise in the charge seven icosahedral Skyrme soliton $[\underline{\underline{9}}]$

\subsection{Interaction energy of two scalar multipoles}

As a technical preparation for the computation of the interaction energy of two Skyrme solitons we derive a formula for the interaction energy of two scalar multipoles. In our description of multipoles we use the conventions of [i1 $\overline{1} \overline{1}]$ throughout. Consider the field of a $2^{M}$-pole centred at $X_{+}=(0,0, R / 2)$, where $R>0$. In terms of spherical coordinates $\left(\theta_{+}, \varphi_{+}\right)$centred at $X_{+}$it reads

$$
f_{M}(x)=\frac{4 \pi}{(2 M+1)} \sum_{m=-M}^{M} Q_{M m} \frac{Y_{M m}\left(\theta_{+}, \varphi_{+}\right)}{\left|x-X_{+}\right|^{M+1}} .
$$

Similarly define $X_{-}=(0,0,-R / 2)$ and let $\left(\theta_{-}, \varphi_{-}\right)$be spherical coordinates centred at $X_{-}$. A $2^{N}$-pole field centred at $X_{-}=(0,0,-R / 2)$ has the form

$$
g_{N}(x)=\frac{4 \pi}{(2 N+1)} \sum_{n=-N}^{N} \tilde{Q}_{N n} \frac{Y_{N n}\left(\theta_{-}, \varphi_{-}\right)}{\left|x-X_{-}\right|^{N+1}} .
$$

A natural measure for the interaction energy between harmonic function in the upper and those in the lower half plane is

$$
V[f, g]=\int_{x_{3}=0} d x_{1} d x_{2}\left(g \partial_{3} f-f \partial_{3} g\right)
$$

The computation of this interaction energy for the multipole fields $f_{M}$ and $g_{N}$ is not easy because the combined field of the two multipoles only has cylindrical symmetry about the $x_{3}$-axis and not the full rotational symmetry of each of the multipoles. As explained in [i] Fourier transformation in the $x_{1} x_{2}$-plane turns out to be an efficient tool for evaluating the 
integral in (2.12). Assuming without loss of generality that $M \leq N$, the answer is

$$
\begin{aligned}
V\left[f_{M}, g_{N}\right]=\frac{(4 \pi)^{2}}{R^{M+N+1}} & \frac{(M+N) !(-1)^{N+M}}{\sqrt{(2 M+1)(2 N+1)}} \times \\
& \sum_{m=-M}^{M} \frac{\bar{Q}_{M m} \tilde{Q}_{N m}}{\sqrt{(M-m) !(M+m) !} \sqrt{(N-m) !(N+m) !}} .
\end{aligned}
$$

For us, the most important feature of the formula (2.13) is that, for non-vanishing multipole moments, the interaction energy can always be made non-zero by a suitable rotation of the multipole of the highest order, in our case $N$ (if $M=N$ it does not matter which of the multipoles gets rotated). By definition the multipole components $\tilde{Q}_{N n}$ are the components of an element $\tilde{Q}$ of the $(2 N+1)$-dimensional irreducible unitary representation $W_{N}$ of $S O(3)$, with $G \in S O(3)$ acting via $\tilde{Q}_{N n} \mapsto \sum_{n^{\prime}=-N}^{N} U_{n n^{\prime}}^{N}(G) \tilde{Q}_{N n^{\prime}}$. Think of the pairing of the multipole moments in (2.13i) as a linear form

$$
F_{Q}: W_{N} \rightarrow \mathbb{R}, \quad \tilde{Q} \mapsto \sum_{m=-M}^{M} \frac{\bar{Q}_{M m} \tilde{Q}_{N m}}{\sqrt{(M-m) !(M+m) !} \sqrt{(N-m) !(N+m) !}} .
$$

Then it follows from the irreducibility of $W_{N}$ that $U^{N}(G) \tilde{Q}$ cannot lie in the kernel of $F_{Q}$ for all $G$. Therefore $F_{Q}\left(U^{N}(G) \tilde{Q}\right) \neq 0$ for some $G$. Hence the interaction energy (2.13) is non-vanishing after rotating $\tilde{Q}$ with that $G$.

\subsection{Interaction energy of well-separated Skyrme solitons}

Consider now two Skyrme solitons $U^{(1)}$ and $U^{(2)}$ of degrees $k$ and $l$. Since the total energies of both $U^{(1)}$ and $U^{(2)}$ are finite there must be balls $B_{1}$ and $B_{2}$ in $\mathbb{R}^{3}$ so that most of the energy of $U^{(1)}$ and $U^{(2)}$ is concentrated in, respectively, $B_{1}$ and $B_{2}$. Outside the balls $B_{1}$ and $B_{2}$ the asymptotic analysis of the previous section applies. Suppose that the leading multipole of $U^{(1)}$ is a $2^{M}$-pole and the leading multipole of $U^{(2)}$ is a $2^{N}$-pole. Denoting the radii of $B_{1}$ and $B_{2}$ by $D_{1}$ and $D_{2}$ we have

$$
U^{(1)}(x) \sim 1+u_{M}(x) \text { for } \quad x \notin B_{1}
$$

and

$$
U^{(2)}(x) \sim 1+v_{N}(x) \quad \text { for } \quad x \notin B_{2},
$$

where $u_{M}$ is of the form $\left(\overline{2}, . \bar{g}^{\prime}\right)$ and analogously

$$
v_{N}(x)=i \tau_{a} \sum_{n=-N}^{N} \frac{4 \pi}{2 N+1} \tilde{Q}_{N n}^{a} \frac{Y_{N n}(\theta, \varphi)}{r^{N+1}} .
$$

Using the translational invariance of the Skyrme energy functional we can assume without loss of generality that $B_{1}$ is centred at $X_{+}=(0,0, R / 2)$ and that $B_{2}$ is centred at $X_{-}=$ $(0,0,-R / 2)$, where $R$ is so large that $B_{1}$ and $B_{2}$ do not overlap, i.e. $R>D_{1}+D_{2}$. The 
parameter $R$ will be interpreted as the separation of the Skyrme solitons. Then we define the following product configuration

$$
U_{R}(x)=U^{(1)}(x) U^{(2)}(x)
$$

This configuration has degree $k+l$ and finite energy, so that $U_{R} \in \mathcal{C}_{k+l}$. A lengthy calculation performed in [i] shows that

$$
E\left[U_{R}\right]=E\left[U^{(1)}\right]+E\left[U^{(2)}\right]+\Delta E+\mathcal{O}\left(\frac{1}{R^{2 N+4}}\right)+\mathcal{O}\left(\frac{1}{R^{2 M+4}}\right),
$$

where

$$
\Delta E=2 \sum_{a=1}^{3} \int_{x_{3}=0} d x_{1} d x_{2}\left(u_{M}^{a} \partial_{3} v_{N}^{a}-v_{N}^{a} \partial_{3} u_{M}^{a}\right) .
$$

Now we note that $\Delta E$ is the sum over iso-components of the the scalar interaction terms we studied above

$$
\Delta E_{a}=2 \int_{x_{3}=0} d x_{1} d x_{2}\left(u_{M}^{a} \partial_{3} v_{N}^{a}-v_{N}^{a} \partial_{3} u_{M}^{a}\right)=-2 V\left[u_{M}^{a}, v_{N}^{a}\right] .
$$

Picking one of the iso-indices, say $a=1$, we can use iso-rotations to make sure that the first iso-components $u_{M}^{1}$ and $v_{N}^{1}$ are non-vanishing. The result of the previous subsection then implies that we can make the multipole interaction energy $\Delta E_{1}$ non-zero by a spatial rotation of the Skyrme soliton with the higher multipole order.

Now consider the sum

$$
\Delta E=\Delta E_{1}+\Delta E_{2}+\Delta E_{3}
$$

Following an idea in [i] $\overline{1}]$ we would like to show that we can always arrange for $\Delta E$ to be negative by a suitable iso-rotation of one of the Skyrme solitons. We may assume that, possibly after re-labelling the pion fields,

$$
\Delta E_{1} \geq \Delta E_{2} \geq \Delta E_{3}
$$

If $\Delta E<0$ we are done, so suppose that $\Delta E \geq 0$. Since we know that not all $\Delta E_{a}$ vanish we can conclude that $\Delta E_{1}>0$. Now perform an iso-rotation of Skyrme soliton 2 by 180 degrees around the third iso-spin axis. This reverses the sign of $v_{N}^{1}$ and $v_{N}^{2}$ and hence of $\Delta E_{1}$ and $\Delta E_{2}$. The new value of $\Delta E$ is

$$
\begin{aligned}
\Delta E & =-\Delta E_{1}-\Delta E_{2}+\Delta E_{3} \\
& =-\Delta E_{1}-\left(\Delta E_{2}-\Delta E_{3}\right)<0,
\end{aligned}
$$

since $-\Delta E_{1}<0$ and, with our ordering, $-\left(\Delta E_{2}-\Delta E_{3}\right) \leq 0$.

Thus, the contribution $\Delta E$ to the interaction energy of two Skyrme solitons $U^{(1)}$ and $U^{(2)}$ can always be made negative by suitable relative rotations and iso-rotations of the Skyrme solitons. Returning to the expression (2.19) for the interaction energy and 
noting from (2.13) that $\Delta E$ falls off like $R^{-(N+M+1)}$, we conclude that $\Delta E$ is the leading contribution for large separation $R$ provided the orders of the leading multipoles do not differ by more than 2 , i.e. provided that $|N-M| \leq 2$. In that case we can therefore always arrange for the interaction energy to be negative for sufficiently large separation. In symbols, for sufficiently large $R$ and the appropriate orientations we have

$$
E\left[U_{R}\right]<E\left[U^{(1)}\right]+E\left[U^{(1)}\right] .
$$

\section{The existence of Skyrme solitons}

The results of the previous section are not sufficient to derive the existence minima in a general sector $\mathcal{C}_{k}$ of the Skyrme model. Nonetheless it is instructive to see how far one can get with the following two extra assumptions.

1. When minima exist they satisfy the technical assumptions made in [i]. In particular the field $U$ and the currents $L_{i}$ are Hölder continuous.

2. The leading multipole of any Skyrme soliton is at most an octupole (order 3).

The second assumption seems particularly restrictive and deserves a comment. Since monopoles (order 0) cannot arise in Skyrme solitons and dipoles (order 1) are known to arise in some, the restriction to leading multipoles of order at most 3 means that any two Skyrme solitons we consider in the following have multipoles whose orders do not differ by more than 2. As mentioned earlier, none of the numerically studied Skyrme soliton violate assumption 2 .

Recall from section 1 that Skyrme solitons have rigorously been shown to exist in the topological sectors $\mathcal{C}_{1}$ and $\mathcal{C}_{-1}$. Although it is not clear whether the minimum is of the hedgehog form (11.11), we can use the numerically computed energy of the Skyrmion to give an upper bound on the infimum $I_{1}$. To avoid the discussion of numerical accuracy we use the energy of the instanton generated hedgehog field [i] $\left[\begin{array}{l}1 \\ \overline{8}\end{array}\right] \tilde{I}_{1}=1.24 \times 12 \pi^{2}$ to estimate

$$
I_{1}<\frac{5}{4} 12 \pi^{2} .
$$

Using Esteban's weak inequality (1, $\left.1 \overline{1} \overline{1}_{1}^{\prime}\right)$ we deduce

$$
I_{k}<\frac{5}{4} 12 \pi^{2}|k|
$$

for all $k \in \mathbb{Z}$. Combining this result with the energy bound (1)

$$
I_{k}<\sum_{s} I_{l_{s}}
$$

for all $k \in \mathbb{Z}$ and $l_{s} \in \mathbb{Z}, s \in \mathbb{N}$ satisfying $\sum_{s} l_{s}=k$ and $\sum_{s}\left|l_{s}\right| \geq \frac{5}{4}|k|$. Thus we can weaken the assumption in Esteban's theorem about the existence of minima. The existence of a minimum in sector $\mathcal{C}_{k}$ follows if the strict inequality (1.13) is satisfied for all $k \in \mathbb{Z}$ and all $l \in \mathbb{Z}-\{k, 0\}$ satisfying

$$
|l|+|k-l|<\frac{5}{4}|k|
$$


Combining this result with our proof of attraction between Skyrme solitons and the assumptions made above we can prove the existence of minima in the sectors $\mathcal{C}_{k}$ for low values of $k$. We prove the claim by induction and first consider positive $k$. We know from [i-i] that the minimum exists for $k=1$. Suppose that minima exist in $\mathcal{C}_{l}$ with $l=1,2, \ldots, k-1$. Then apply the product ansatz $U_{R}$ and the inequality (2.25) to pairs of Skyrme solitons $U^{(1)}$ and $U^{(2)}$ of degree $l$ and $k-l$ for $l=1, \ldots, k-1$. By assumption the energies of these Skyrme solitons are equal to the infima $I_{l}$ and $I_{k-l}$. Then, by definition of the infimum $I_{k}$ $(1,10 i)$ and from $(2.25 i)$

$$
I_{k} \leq E\left[U_{R}\right]<E\left[U^{(1)}\right]+E\left[U^{(1)}\right]=I_{l}+I_{(k-l)}
$$

for all $l=1, \ldots, k-1$. As long as $k \leq 8$ the only integers $l$ satisfying the inequality ( $(\overline{3}-4)$ are in the range $l=1, \ldots, k-1$, so that we have satisfied the condition of Esteban's theorem and can conclude that the infimum in $\mathcal{C}_{k}$ is attained. The claim for negative $k$ follows by applying the energy preserving reflection map $U(x) \mapsto U^{\dagger}(-x)$.

For $k>8$ the step in the inductive proof fails because we then need to consider the

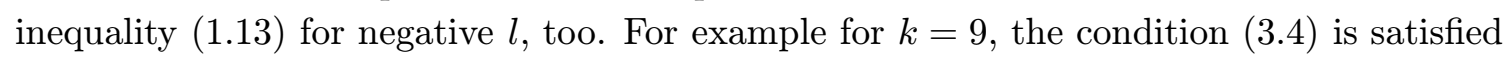
for $l=-1$ and $k-l=10$. However, since we do not know whether Skyrme solitons exist in the sector $\mathcal{C}_{10}$ we cannot establish the inequality $I_{9}<I_{-1}+I_{10}$ with our method. Numerical evidence and physical intuition suggests that the inequality (1.13i) should certainly hold when $k$ is positive and $l$ or $(k-l)$ is negative. If it was violated in that range it would be energetically favourable for matter (solitons of positive degree) and antimatter (solitons of negative degree) to coexist in some sectors of the Skyrme model. However, while this seems unlikely, we cannot disprove the possibility with our methods. If one could rule it out by other methods, the inductive argument given above could be extended to prove the existence of minima in all sectors of the Skyrme model.

To end, I point out that it seems plausible that our results can also be used to give a more direct proof of the existence of minima in all sectors of the Skyrme model. The idea of such a proof is that the infimum in one of the sectors, say $\mathcal{C}_{k}$, can only fail to be attained if it is energetically favourable for configurations in that sector to split into well-separated Skyrme solitons. The infimum $I_{k}$ would then be realised by a virtual solution, made up of infinitely separated Skyrme solitons. The number of Skyrme solitons in this virtual solution is necessarily finite. For suppose the degrees of the Skyrme solitons are $l_{s}, s \in \mathbb{N}$ with $\sum_{s} l_{s}=k$. By the inequality $(\underline{3} \cdot \overline{3} \cdot \bar{j})$ we can only have the equality

$$
I_{k}=\sum_{s} I_{l_{s}}
$$

if $\sum_{s}\left|l_{s}\right|<\frac{5}{4}|k|$, which means that all but a finite number of the $l_{s}$ must be zero. Suppose that $l_{s} \neq 0$ for $s=1,2 \ldots S$ and $l_{s}=0$ for $s>S$. Physically, one expects the following dichotomy to hold.

(a) For given $k$ the infimum $I_{k}$ is attained by a Skyrme soliton of degree $k$. 
(b) The infimum $I_{k}$ is equal to a finite sum

$$
I_{k}=\sum_{s=1}^{S} E_{l_{s}}
$$

of energies $E_{l_{s}}=I_{l_{s}}$ of $S$ Skyrme solitons of degrees $l_{s} \in \mathbb{Z}-\{0, k\}$.

With our assumption on the leading multipoles our earlier proof of attractive forces in the Skyrme model implies that we can always lower the energy of a finite number of infinitely separated Skyrme solitons by chosing appropriate orientations for two of the Skyrme solitons and bringing them closer together. Thus the infimum $I_{k}$ cannot be of the form $\left(\overline{3}_{-} \bar{z}_{1}\right)$ and if the dichotomy is valid, option (a) must hold.

\section{Conclusion}

In this talk I reviewed the result, obtained jointly with Manton and Singer, that arbitrary Skyrme solitons attract for suitable relative orientation and iso-orientation, provided the orders of their leading multipole moments do not differ by more than two. I also indicated how this result, combined with the work of Esteban, can be used to shed light on the question of minima in the Skyrme model. However, the approaches sketched here also show how difficult it is to establish rigorous results on minima. In particular the assumption on the multipoles of Skyrme solitons severely restricts the generality of the sort of argument outlined above.

\section{Acknowledgments}

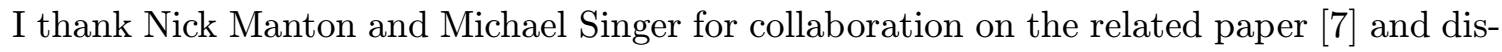
cussions. I acknowledge financial support trough an EPSRC advanced research fellowship.

\section{References}

[1] T. H. R. Skyrme, A non-linear field theory, Proc. Roy. Soc. A 260 (1961) 127-138.

[2] M. J. Esteban and S. Müller, Soboblev maps with integer degree and applications to Skyrme's problem, Proc. Roy. Soc. London A 436 (1992) 197-201.

[3] L. D. Faddeev, Some comments on the many-dimensional solitons, Lett. Math. Phys. 1 (1976) 289-293.

[4] N. S. Manton, Geometry of Skyrmions, Comm. Math. Phys. 111 (1987) 469-478.

[5] L. B. Kapitanski and O. A. Ladyzenskaia, On the Coleman's principle concerning the stationary points of invariant functionals, Zap. Nauchn. Semin, LOMI 127 (1982) 84-102.

[6] M. J. Esteban, A direct variational approach for Skyrme's model for meson fields, Comm. Math. Phys. 105 (1986) 571-591.

[7] N. S. Manton, B. J. Schroers and M. A. Singer, The interaction energy of well-separated Skyrme solitons, EMPG-02-22, DAMTP-2002-142, hep-th/0212075 
[8] E. Braaten, L. Carson and S. Townsend, Novel structure of static multisoliton solutions in the Skyrme model, Phys. Lett. 235 B (1990) 147-152.

[9] R. A. Battye and P. M. Sutcliffe, Symmetric Skyrmions, Phys. Rev. Lett. 79 (1997) 363-366.

[10] R. A. Battye and P. M. Sutcliffe, Skyrmions, fullerenes and rational maps, Rev. Math. Phys. 14 (2002) 29-86.

[11] R. A. Leese, N. S. Manton and B. J. Schroers, Attractive channel Skyrmions and the deuteron, Nucl. Phys. B 442 (1995) 228-267.

[12] R. B. Melrose, The Atiyah-Patodi-Singer Index Theorem, Research Notes in Mathematics 4, A. K. Peters Ltd, Wellesley 1993.

[13] R. R. Mazzeo, Elliptic theory of differential edge operators I, Comm. Partial Differential Equations 16 (1991) 1615-1664.

[14] L. Hörmander, The analysis of linear partial differential operators III, Grundlehren der mathematischen Wissenschaften 274, Springer Verlag 1985.

[15] J. D. Jackson, Classical Electrodynamics, Wiley 1972.

[16] L. Castillejo and M. Kugler, The interaction of Skyrmions, unpublished preprint, 1987.

[17] N. S. Manton, Skyrmions and their multipole moments, Acta Phys. Polonica B 25 (1994) 1757-1764.

[18] M. F. Atiyah and N. S. Manton, Skyrmions from Instantons, Phys. Lett. 222B (1989) 438-442. 